\title{
e-Migrinter
}

$10 \mid 2013$

Genre et imbrication des rapports de domination dans les médias des minorités ethniques

\section{Tracing Social inequalities in Environementally-induced} Migration

Colloque international de Bielefeld, du 9 au 13 décembre 2012

Émilie Chevalier et Eleonora Guadagno

\section{OpenEdition}

\section{Journals}

Édition électronique

URL : https://journals.openedition.org/e-migrinter/446

DOI : 10.4000/e-migrinter.446

ISSN : 1961-9685

Éditeur

UMR 7301 - Migrinter

Édition imprimée

Date de publication : 11 avril 2013

Pagination : 86-90

ISSN : 1961-9685

Référence électronique

Émilie Chevalier et Eleonora Guadagno, «Tracing Social inequalities in Environementally-induced Migration », e-Migrinter [En ligne], 10 | 2013, mis en ligne le, consulté le 20 mai 2021. URL : http:// journals.openedition.org/e-migrinter/446 ; DOI : https://doi.org/10.4000/e-migrinter.446 


\title{
"Tracing Social inequalities in Environementally-induced Migration ", Colloque international, Bielefeld, du 9 au 13 décembre 2012
}

\author{
Compte-rendu par Émilie Chevalier \& Eleonora Guadagno
}

La conférence a également comporté deux séances de présentation de posters scientifiques. La liste de ces derniers, ainsi que le programme complet, peuvent être consultés à l'adresse suivante : $\langle$ http://www.esf.org/activities/esf-conferences/details/2012/confdetail402.html >

D

u 9 au 13 décembre derniers, s'est

tenu, à l'Université de Bielefeld, dans le Zentrum für interdisziplinäre Forschung, en Allemagne, un colloque international organisé conjointement par l'European Science Foundation, le Gicc-Exclim ${ }^{1}$, et $C_{C S} T^{2}$. Trois membres de Migrinter ont participé à ce colloque : Émilie Chevalier et Eleonora Guadagno ont présenté des posters, (le premier nommé Factoring climate variabilities and changes in migration decision-making : a socially and spatially discriminating process et le second The green revolution and the impact of indigenous populations ), Mme Véronique Lassailly-Jacob, dans la session portant sur la gestion des déplacements des populations dues aux phénomènes climatiques extrêmes, a présenté une intervention intitulée Social and spatial inequalities linked to

flood-induced displacement in Burkina Faso. Pendant trois jours, les questions relatives aux liens entre les dégradations de l'environnement, les inégalités, la vulnérabilité et les mobilités humaines ont été abordées. Plusieurs institutions académiques et organisations internationales, telles que les Nations Unies ou différentes Universités de nombreux pays, ont apporté leur contribution à cette importante rencontre scientifique pluridisciplinaire et internationale.

${ }^{1}$ Gérer les déplacements des populations dues aux phénomènes climatiques extrêmes.

${ }^{2}$ European Cooperation in Science and Technology.
Thomas Faist et Janette Shade, de l'Université de Bielefeld, ont ouvert la discussion rassemblant de nombreux géographes, anthropologues, politologues et sociologues pour interroger et tenter de décrypter les liens entre inégalités/égalités et catastrophes naturelles, avec un regard spécifique porté sur la mobilité précédant et suivant l'événement catastrophique, dans une volonté commune de faire évoluer ce champ de recherche.

Le colloque a débuté avec une première session, le lundi 10 décembre, présentée par Kerstin Schmidt-Verkel, de l'International Council for Science, qui avait comme objectif d'analyser le rôle des théories migratoires dans l'appréhension des inégalités sociales liées aux dégradations environnementales. Ahmet Icduygu, de l'Université de Koç (Turquie ) a attiré l'attention sur le fait que les théories migratoires ont toujours été descriptives plutôt qu'explicatives - en laissant de côté la dimension «environnemental » des migrations n'entrant elle que rarement en compte dans les schémas interprétatifs traditionnels. Selon la démographe, il est donc nécessaire de mettre en place un nouveau cadre théorique qui puisse, dans ce domaine, considérer la migration en intégrant différents niveaux d'analyse (macro, micro et meso). La deuxième intervention a été celle de Haydea Izazola, de l'Université de Xochimilco (Mexique), qui a présenté un cas d'étude sur les inégalités provoquées par les migrations dans le contexte général du Mexique et celui, plus particulier, de l'État de Tabasco: selon la 
chercheuse, seules les classes aisées auraient eu l'opportunité de pouvoir s'éloigner des zones les plus gravement touchées par la dégradation environnementale. Ensuite, Pilesjo Petter et Lina Eklund, de l'Université de Lund (Suède), ont présenté une autre étude de cas concernant les implications pour les populations rurales dans la région de Duhok en Iraq, à travers l'utilisation du système SIG (Système d'Information Géographique): ils ont mis en évidence comment, dans une région pourtant touchée par une forte sécheresse, la migration n'est pas utilisée comme stratégie adaptative par les communautés y vivant. La dernière intervention de la session, focalisée sur le concept de «translocalité » (c'est à dire l'ensemble des réseaux sociaux et culturels qui sont entretenus par les personnes et lieux) comme angle d'analyse pour la migration environnementale, a été présenté par Patrick Sakdaporlak, de l'Université de Bonn (Allemagne), avec une recherche de terrain au Kenya, analysée par Clemens Grenier de la même Université de Bonn. Pour finir, Yan Tan, de l'Université d'Adelaïde (Australie), a questionné le phénomène de l'inégalité et de la dégradation environnementale dans les deux districts chinois de Shanghai et Nanjing, relevant comment, même en milieu urbain, la dégradation environnementale peut provoquer la mobilité. Son intervention a particulièrement insisté sur l'aspect méthodologique, notamment en ce qui concerne les entretiens avec les familles.

La deuxième session de la journée, présidée par Carsten Felgentreff de l'Université de Osnabruk (Allemagne) et Andrew Baldwin, de l'Université de Durham (Grande Bretagne), s'est focalisée sur les inégalités sociales comme vecteurs des migrations environnementales. À travers ce cadre théorique, ont été présentés plusieurs cas d'études dans différents contextes géographiques. Tout d'abord, Tamer Afifi, de l'Université des Nations Unies, a présenté le projet Rainfall, financé par l'ONU, qui opère dans différents pays pour tracer les liens entre le changement des régimes des précipitations, la sécurité alimentaire et la mobilité humaine. Un de ces cas d'étude a donc été présenté comme modèle opérationnel par Benjamin Etzold, de l'Université de Bonn qui a analysé les changements environnementaux liés aux pluies, aux inondations et à la sécheresse au Bangladesh. Ensuite, toujours en ce qui concerne le Bangladesh, Architesh Panda, de l'Indian Institute for Social and Economic Change a présenté un cas d'étude sur les conflits frontaliers entre le nord de l'Inde et le Bangladesh qui seraient causés par des raisons environnementales et par un manque de ressources alimentaires et agricoles. Sur ce point, en argumentant que le facteur environnemental est une cause de conflit et de vulnérabilité, Charles Anemone et Iwebunor Okwechime ont présenté deux cas d'études concernant les conflits entre les nomades et les communautés agricoles dans le couloir des éleveurs en Ouganda à cause de la perte de terrains fertiles et des conflits issus de l'économie du pétrole dans le Delta du Niger. Les deux cas d'études montrent comment les inégalités économiques peuvent avoir des effets pervers sur les migrations et les relations sociales entre communautés.

La troisième session, dirigée par Janette Shade, a été centrée sur les mécanismes de reproduction des inégalités en contexte migratoire. La première intervention de Kai Achmidt-Sotau de l'institut suisse Science Solution a montré comment le processus de réinstallation des communautés déplacées peut augmenter les inégalités économiques et provoquer à la fois la marginalité sociale et la vulnérabilité des individus. Ensuite, Eberhard Weber, de l'Université de Suva (Fiii), a analysé la réinstallation de différentes communautés touchées par le tsunami de 2005 et a noté comment cette réinstallation a produit des inégalités. Selon le chercheur, les changements climatiques et une montée des eaux conséquente pourraient endommager encore davantage les îles Fiji, provoquant encore 
des migrations et le besoin de réinstallation des populations. Toujours en ce qui concerne l'hémisphère sud, Gil Marvel Tabucanon, de l'Université de Macquaire (Australie), a souligné le cas des habitants de l'île Nauru, qui auraient dû être déplacés en Australie, mais qui ont refusé pour pouvoir garder leur indépendance et l'accès aux ressources de l'île. François Gemenne, de l'IDDRI de Paris, a analysé le cas du tsunami de 2009 au Japon, qui a provoqué la destruction de la centrale nucléaire de Fukushima: pour le chercheur, les conséquences de ce désastre montrent que, même dans un pays au capitalisme avancé, la gestion des catastrophes naturelles reste extrêmement délicate. En effet, on se trouve ici face à une population victime des radiations qui a subi un traitement injuste et inéquitable par rapport aux déplacés du tsunami même (et non irradiés) pour lesquels étaient déjà en place un système d'alerte et un système juridique qui ont permis une réinstallation immédiate. Pour finir, Bishawjit Malik, du Karlsrube Institute of Technology (Allemagne), a relevé comment les cyclones au Bangladesh provoquent des déplacements qui augmentent le "landgrabbing ", la dégradation environnementale et la corruption économique.

La journée a été conclue par Thomas Faist qui a fait le point en analysant de quelle manière le politique, l'écologie et la société sont liés et dans quelle mesure le phénomène migratoire contient et amplifie les liens entre ces éléments. Le chercheur a, en outre, ouvert le débat du mardi 11 décembre, en présentant une session portant sur l'analyse de la conceptualisation de l' "inégalité » dans un contexte de migrations environnementales.

Oliver Backwell, de l'Université d'Oxford (Royaume-Uni), a porté l'attention sur l'inapplicabilité des catégories conceptuelles utilisées jusqu'à maintenant dans le domaine des recherches en Sciences humaines, du fait qu'elles sont extrêmement orientées politiquement mais peu efficaces pour la protection des migrants. L'idée que l'identité des communautés et l'inégalité entre les individus soient modifiées par la perception et les conséquences des désastres a été analysée par Fatima Yamin, de l'Université d'Islamabad (Pakistan), laquelle a étudié le rôle de la perception du risque dans la formation des identités communautaires au Pakistan. De plus, Alexandra Winkels, de l'Université de West Anglia (RoyaumeUni), a noté comment, souvent, les migrants transfèrent leur vulnérabilité de la zone de départ à celle de destination, quittant des zones dangereuses pour s'installer dans des zones qui les exposent plus encore à des situations de risque. La dernière intervention de la session a été celle de Robert McLeman, de l'Université Wilfrid Laurier (Californie) : le chercheur a mis en évidence comment la migration peut être issue d'un ensemble de causes climatiques, environnementales et alimentaires. Les effets de la dégradation environnementale peuvent aussi bien causer une mobilité des pays du Sud, qu'une mobilité à partir des communautés des pays du Nord en quête d'un climat meilleur et d'un environnement moins dégradé.

Les trois dernières sessions du colloque se sont tenues le mercredi 12 décembre. La première a été consacrée aux liens entre genre et inégalités sociales en matière de migrations environnementales. En ouverture de cette cinquième session, Brooke A. Ackelry, de l'Université américaine Vanderbilt, a présenté une synthèse des cadres théoriques et éthiques pouvant servir de référence pour étudier les inégalités entre sexes dans un contexte de changements environnementaux. Après avoir rappelé différentes théories de la justice, l'auteur a notamment insisté sur les difficultés méthodologiques associées à l'appréhension des inégalités entre sexes, du fait de leur «invisibilité » (inégalités fréquentes et familières et donc ancrées dans les représentations quotidiennes - phénomène en apparence fragmenté et limité en volume). En dépit de ces contraintes métho- 
dologiques, B. A. Ackelry a toutefois insisté sur l'impératif moral de la prise en compte de ces différences, en soulignant notamment le lien entre égalité des sexes et résilience face aux changements environnementaux.

Trois études de cas ont suivi cette présentation. Dans un premier temps, Victoria van der Land, de l'Institute for Social Ecological Research (Allemagne), a évoqué des recherches menées au Mali et au Sénégal. Les résultats empiriques ont montré qu'au sein de communautés agricoles sahéliennes vulnérables face aux changements environnementaux, les migrations peuvent constituer une stratégie d'adaptation: dans ce contexte, les différences de genre influencent les processus de décision migratoire à travers les attentes associées à la mobilité, mais également les capacités à migrer, l'acceptation sociale de la migration particulièrement dans le cas féminin. Dans l'intervention suivante, Kerstin Schmidt-Verkerk, du Bureau régional pour l'Amérique latine et la Caraỉbe de l'International Council for Science, a posé la question du rôle joué par les inégalités sociales, et plus particulièrement par les inégalités hommes-femmes, dans les choix migratoires associés au changement climatique au Mexique: d'après les résultats d'enquêtes menées à Veracruz et Zacatecas, l'auteur affirme que la migration peut constituer une stratégie d'adaptation face aux contraintes climatiques. Toutefois, le recours à la migration n'est possible que pour les individus disposant d'un certain niveau de ressources et de réseaux sociaux. Au sein de ces inégalités sociales, sont à noter des inégalités de genre. En effet, si la migration féminine est commune à Veracruz, elle ne l'est pas à Zacatecas car considérée comme «inappropriée et dangereuse ». Dans la troisième présentation, Mizanur Rahman montre que face aux changements climatiques, des femmes et filles de paysans du sud du Bangladesh émigrent vers des grandes villes indiennes où elles occupent des emplois domestiques. L'auteur souligne que ce phénomène concerne plus particulièrement des familles qui n'ont pas pu mobiliser suffisamment de ressources pour envoyer l'homme travailler dans les pays d'Asie demandeurs de main d'œuvre.

La présentation du projet Exclim a fait l'objet de la session suivante. Chloé Vlassopoulos, de l'Université de Picardie (France), a proposé une description synthétique du projet. Ces recherches ont consisté en une analyse des acteurs impliqués dans ces questions au niveau international et des initiatives existantes, ainsi qu'à une étude de l'adaptabilité des populations et des réponses institutionnelles face aux phénomènes climatiques extrêmes. Mme Vlassopoulos a notamment souligné la diversité des angles d'approche et des objectifs mis en œuvre par les institutions, ainsi que le manque de politiques coordonnées et trans-sectorielles à même de prendre en compte la complexité des dégradations environnementales et d'améliorer la situation initiale des populations et l'importance de la participation des populations dans les politiques de gestion territoriale. Véronique Lassailly-Jacob, membre du laboratoire Migrinter (Université de Poitiers), a ensuite présenté le bilan de recherches de terrain portant sur les conditions des populations déplacées à la suite d'inondations au Burkina Faso. Il a été noté que les déplacements organisés par les autorités ont contribué au renforcement des inégalités sociales pour deux raisons: d'une part, l'aide n'a pas été allouée à tous les déplacés mais seulement à certaines catégories de populations (individus propriétaires d'une maison détruite par exemple), d'autre part, le choix d'un site éloigné de Ouagadougou pour la réinstallation des populations a engendré pour celles-ci des difficultés d'accès à l'emploi. Dans le cas de la Turquie, Gulcin Erdi Lelandais de l'Université de Rouen, a souligné l'implication récente du pays dans les politiques multilatérales en matière de changement climatique. Toutefois, son analyse des liens entre la désertification et les migrations des agriculteurs dans la plaine de Konya a montré que si le changement climatique pouvait constituer un outil d'insertion 
politique internationale pour le pays, les engagements internationaux n'étaient pas associés à une réelle mise en œuvre de politiques internes. David Méchin (Université de Reims) et Sylvia Bruzzone (Université de Picardie - CURAPP/CNRS) ont conclu ce panel par deux présentations respectivement consacrées à la gestion des impacts de l'ouragan Katrina à La Nouvelle-Orléans et aux liens entre déplacements environnementaux et gouvernance des risques.

L'ultime session de la conférence a porté sur les enjeux de gouvernance associés aux migrations environnementales. Dans un premier temps, Elisa Fornalé et Jérémie Guélat (Universités de Berne et de Neuchâtel) se sont concentrés sur l'enjeu particulier de l'apatridie climatique dans le contexte de la disparition d'États insulaires envisagé comme conséquence de la hausse du niveau marin. Après avoir réalisé un état des lieux des territoires exposés à ce risque à travers le monde, les auteurs ont proposé un certain nombre de solutions susceptibles d'être mises en œuvre afin de garantir aux populations concernées le maintien de leur droit à une nationalité, composante de la Déclaration universelle des Droits de l'Homme. Dans un second temps, Benoît Mayer, doctorant en droit de l'Université nationale de Singapour, a offert une perspective critique sur la construction du concept de réfugié climatique. L'auteur a d'abord montré qu'une analyse des théories de la justice conduisait à mettre en question la légitimité de la création d'une catégorie légale spécifiquement orientée vers des migrants climatiques. Il a en effet souligné la fragilité des fondements éthiques d'une telle initiative. B. Mayer a par ailleurs soulevé la question des possibles utilisations politiques de la notion de réfugié climatique. La troisième présentation, effectuée par Andrew Baldwin (Université de Durham, Grande-Bretagne), a porté sur la racialisation de la notion de migrant climatique. S'appuyant sur des analyses de Michel Foucault, A. Baldwin a cherché à montrer que l'accent mis sur les pays du Sud en ma- tière de migrations climatiques pouvait relever d'un discours racial et d'une recherche de contrôle des populations circulantes.

La clôture de la conférence a été effectuée par Jeanette Schade et Thomas Faist. Ce dernier a effectué un bilan des présentations orales et a constaté que se dégageaient quatre types de réflexions: l'étude des mécanismes sociaux à l'œuvre en matière de migrations environnementales; les travaux portant sur les concepts de vulnérabilité, d'adaptation, de résilience; les réflexions normatives sur l'égalité en matière environnementales; enfin, les questionnements plus spécifiques comme celui de l'apatridie climatique. Celui-ci a également souligné que l'un des enjeux clé des politiques d'adaptation face aux enjeux environnementaux résidait dans l'atténuation des facteurs de vulnérabilité au niveau local. À l'issue de ces interventions, une ultime présentation a été effectuée par John Urry, de l'Université de Lancaster. Cette réflexion a dépassé le seul cadre des inégalités sociales en matière de migrations environnementales. L'idée centrale soutenue par l'auteur était que la prévention des changements environnementaux ne pourrait être effective qu'à travers des innovations permettant l'avènement d'un nouveau système social et économique qui rendrait le système actuel socialement inégal et prédateur de ressources - obsolète.

Émilie Chevalier Doctorante en Géographie GEOLAB / Université de Limoges MIGRINTER - UMR 7301 CNRS / Université de Poitiers emilie.chevalier1@gmail.com

Eleonora Guadagno

Doctorante en Géographie MIGRINTER - UMR 7301 CNRS / Université de Poitiers guadagno.eleonora@gmail.com 